\title{
A Weibull Generated Exponentiated Exponential Model: Properties and Bayes Interval Prediction
}

\author{
Essam K. AL-Hussaini \\ Department of Mathematics, Alexandria University, Egypt \\ ekalh2014@yahoo.com \\ Alaa H. Abdel-Hamid \\ Department of Mathematics and Computer Science \\ Beni-Suef University, Egypt \\ hamid_alh@science.bsu.edu.eg
}

Received 21 August 2014

Accepted 20 May 2015

\begin{abstract}
Motivated by the fact that the so-called Weibull generated exponentiated exponential distribution (WGEED) accommodates for non-monotone as well as monotone hazard rate functions (HRFs), we give some properties of this WGEED and explore its use in life testing by obtaining Bayes prediction intervals of future observables. The survival function (SF) of the WGEED is constructed by composing a Weibull cumulative distribution function (CDF) with $-\ln$ [exponentiated exponential] CDF. Some properties of the WGEED are given and prediction intervals of future observables, using the one- and two-sample schemes, are obtained. A comparison between the WGEE and the Weibull distributions, based on Kolmogorov-Smirnov goodness of fit test, shows that the former fits better than the latter. Real life data shows the possibility of using the WGEED in analyzing lifetime data. Numerical examples of one- and two- sample Bayes interval prediction are given to illustrate the procedure and a simulation study is made to compute the coverage probabilities and the average lengths of intervals.
\end{abstract}

Key words: Composed distributions; Weibull and exponentiated exponential distributions; one- and two-sample Bayes interval prediction; simulation.

2000 Mathematics Subject Classification: 60E05; 62N01; 62F15

\section{Introduction}

New families of distributions that accommodate for non-monotone as well as monotone hazard rate functions (HRFs) can be constructed by the composition of a given cumulative distribution function (CDF), say $H($.$) ,$ with another CDF $G($.$) or a function of G($.$) . For example$

$$
F(x)=H(G(x))
$$

or

$$
\bar{F}(x)=H(-\ln G(x))
$$


where $\bar{F}(x)=1-F(x)$, is the SF of $F$, produce new families of distributions. If $H(x)$ is positive on the unit interval, then Eq. (1) could be written as

$$
F(x)=\int_{0}^{G(x)} h(y) d y
$$

where $h($.$) is the probability density function (PDF) corresponding to the absolutely continuous CDF H($.). Eugene et al [1] chose the beta $(\alpha, \beta)$ density for $h(y)$ and the normal CDF for $G$, so that

$$
F(x)=\int_{0}^{G(x)} \frac{1}{B(\alpha, \beta)} y^{\alpha-1}(1-y)^{\beta-1} d y .
$$

Jones [9], generalized $G$ to be an arbitrary CDF (including the normal CDF).

For an arbitrary $G, \operatorname{CDF}(4)$ is known as the beta-G distribution.

Distributions obtained by composition of this kind, such as the beta-normal, beta-Fréchet, beta-Gumbel, beta-exponential, beta-exponentiated exponential, beta- Burr type XII, beta-Weibull and beta power distributions, were studied by the authors of references [1]-[8], respectively.

In composition (2), $H($.$) is assumed to be positive on (0, \infty)$. By choosing $H($.$) to be gamma with$ parameters $(\gamma, \delta)$ and $G($.) to be exponentiated exponential with parameters $(\alpha, \beta)$, given by

$$
H(x)=\int_{0}^{x} \frac{\gamma^{\delta}}{\Gamma(\delta)} y^{\delta-1} e^{-\gamma y} d y \text { and } G(x)=\left(1-e^{-\beta x}\right)^{\alpha}, x>0,(\alpha, \beta>0) .
$$

The expression, given by composition (2), can be written as

$$
\bar{F}(x)=H(-\ln G(x))=H\left(-\ln \left(1-e^{-\beta x}\right)^{\alpha}\right)=\int_{0}^{-\alpha \ln \left(1-e^{-\beta x}\right)} \frac{\gamma^{\delta}}{\Gamma(\delta)} y^{\delta-1} e^{-\gamma y} d y .
$$

Ristić and Balakrishnan [10] called $F(x)=1-\bar{F}(x)$, (with $\gamma=1$ ), gamma generated exponentiated exponential distribution.

Suppose that, instead of using the gamma CDF for $H($.$) , we use the Weibull (\eta, \gamma) \mathrm{CDF}$, given by

$$
H(x)=1-e^{-\eta x^{\gamma}}, x>0,(\eta, \gamma>0) .
$$

The composition of $H($.$) with -\ln G($.$) , where H$ and $G$ are given by (6) and (5), respectively, leads to the SF

$$
\bar{F}(x)=H(-\ln G(x))=H\left(-\alpha \ln \left(1-e^{-\beta x}\right)\right)=1-e^{-\eta\left\{-\alpha \ln \left(1-e^{-\beta x}\right)\right\}^{y}} .
$$

So that

$$
F(x)=1-\bar{F}(x)=\exp \left[-\delta\left\{-\ln \left(1-e^{-\beta x}\right)\right\}^{\gamma}\right], x>0,(\delta, \gamma, \beta>0),
$$

where $\delta=\eta \alpha^{\gamma}$.

The CDF, given by (7), shall be called Weibull generated exponentiated exponential (WGEE) distribution with parameters $(\delta, \gamma, \beta)$.

The corresponding PDF is given by

$$
f(x)=\delta \gamma \beta\left\{-\ln \left(1-e^{-\beta x}\right)\right\}^{\gamma-1}\left(\frac{e^{-\beta x}}{1-e^{-\beta x}}\right) \exp \left[-\delta\left\{-\ln \left(1-e^{-\beta x}\right)\right\}^{\gamma}\right] .
$$

The CDF (7) and PDF (8) can be written in the simpler forms 


$$
F(x)=\exp \left[-\delta w^{\gamma}\right]
$$

and

$$
f(x)=\delta \gamma \beta w^{\gamma-1}\left(e^{w}-1\right) \exp \left[-\delta w^{\gamma}\right]
$$

where

$$
w=-\ln \left(1-e^{-\beta x}\right) .
$$

The rest of the paper is organized as follows: Some properties of the model are given in Section 2. Oneand two-sample schemes are used to obtain Bayes prediction intervals in Sections 3 and 4, respectively. Real data and numerical examples followed by some concluding remarks are considered in Section 5.

\section{Some Properties of the WGEE Model}

\subsection{Special cases and related distributions}

(i) If $\gamma=1$, in Eq. (7), the CDF reduces to that of the exponentiated exponential distribution.

(ii) If $X \sim \operatorname{WGEE}(\delta, \gamma, \beta)$ and $W=-\ln \left(1-e^{-\beta X}\right)$, then $W \sim \operatorname{Weibull}(\delta, \gamma)$.

Notice that

$$
F_{W}(w)=P\left[-\ln \left(1-e^{-\beta X}\right) \leq w\right]=1-F_{X}\left[-\frac{1}{\beta} \ln \left(1-e^{-w}\right)\right] .
$$

Using the CDF, given by (7), it follows that $F_{W}(w)=1-e^{-\delta w^{\gamma}}, w>0$, which is the CDF of the Weibull $(\delta, \gamma)$ distribution.

(iii) If $X \sim \operatorname{WGEE}(\delta, \gamma, \beta)$ and $W=\left\{-\ln \left(1-e^{-\beta X}\right)\right\}^{\gamma}$, then $W \sim \operatorname{Exponential}(\delta)$.

2.2. $\ell^{\text {th }}$ Moment: is given, for $\ell=1,2, \ldots$, by

$$
E\left(X^{\ell}\right)=\int_{0}^{\infty} x^{\ell} f(x) d x=\int_{0}^{\infty} x^{\ell} \delta \gamma \beta w^{\gamma-1}\left(e^{w}-1\right) \exp \left[-\delta w^{\gamma}\right] d x .
$$

By applying the transformation $z=\delta w^{\gamma}$, where $w=-\ln \left(1-e^{-\beta x}\right)$, we obtain

$$
E\left(X^{\ell}\right)=\int_{0}^{\infty}\left[-\frac{1}{\beta} \ln \left\{1-e^{-(z / \delta)^{1 / \gamma}}\right\}\right]^{\ell} e^{-z} d z .
$$

2.3. $q^{\text {th }}$ Quantile $x_{q}$ is given, from (9) and (11), by

$$
\begin{aligned}
& x_{q}=F^{-1}(q)=-\frac{1}{\beta} \ln \left[1-\exp \left\{-\left(-\frac{\ln q}{\delta}\right)\right\}^{1 / \gamma}\right] . \\
& \Rightarrow \quad \text { median }=x_{1 / 2}=-\frac{1}{\beta} \ln \left[1-\exp \left\{-\left(\frac{\ln 2}{\delta}\right)\right\}^{1 / \gamma}\right] .
\end{aligned}
$$




\subsection{Mode}

The mode $x$ satisfies the equation $0=f^{\prime}(x)$, provided that $f^{\prime \prime}(x)<0$. It can be shown, after some algebraic manipulations, that the mode $x$ satisfies the equation

$$
x=\ln \left[\frac{\gamma\left(\delta w^{\gamma}-1\right)-1}{w}\right]^{1 / \beta}, \gamma\left(\delta w^{\gamma}-1\right)>1 .
$$

where $w$ is given by (11).

\subsection{HRF and PRHRF}

The hazard rate function (HRF) $\lambda_{F}(x)$ and proportional reversed hazard rate function (PRHRF) $\lambda_{F}^{*}(x)$ are given, from (7) and (8), by

$$
\begin{gathered}
\lambda_{F}(x)=\frac{f(x)}{1-F(x)}=\frac{\delta \gamma \beta w^{\gamma-1}\left(e^{w}-1\right)}{e^{\delta w^{\gamma}}-1}, \\
\lambda_{F}^{*}(x)=\frac{f(x)}{F(x)}=\delta \gamma \beta w^{\gamma-1}\left(e^{w}-1\right),
\end{gathered}
$$

where $w$ is given by (11).

AL-Hussaini and Hussein [11] showed, in general, that any CDF $F$ can be written in terms of the HRF $\lambda_{F}(x)$ and PRHRF $\lambda_{F}^{*}(x)$, as follows

$$
F(x)=\frac{\lambda_{F}(x)}{\lambda_{F}(x)+\lambda_{F}^{*}(x)} .
$$

Equivalently, the SF of $F$ is given by

$$
\bar{F}(x)=\frac{\lambda_{F}^{*}(x)}{\lambda_{F}(x)+\lambda_{F}^{*}(x)}
$$

and the $\operatorname{PDF} f$ is given by

$$
f(x)=\frac{\lambda_{F}(x) \lambda_{F}^{*}(x)}{\lambda_{F}(x)+\lambda_{F}^{*}(x)} .
$$

The HRF (16) takes several shapes for different values of the parameters. For example, the following cases are shown in Figs. 1-5.

$\begin{array}{llll}\text { Case a: } & (\delta=0.2, \gamma=0.7, \beta=3) & \Rightarrow & \text { decreasing HRF (DHRF) } \\ \text { Case b: } & (\delta=5, \gamma=0.7, \beta=0.5) & \Rightarrow & \text { increasing HRF (IHRF) } \\ \text { Case c: } & (\delta=1, \gamma=0.7, \beta=4.5) & \Rightarrow & \text { decreasing -increasing HRF (DIHRF) } \\ \text { Case d: } & (\delta=1.8, \gamma=0.7, \beta=0.1) & \Rightarrow & \text { bathtub HRF (BTHRF) } \\ \text { Case e: } & (\delta=0.4, \gamma=12.7, \beta=1.2) & \Rightarrow & \text { upside down bathtub HRF (UBTHRF) }\end{array}$ 
Remark: A constant HRF is achieved if, in (16) $\gamma=\delta=1$.
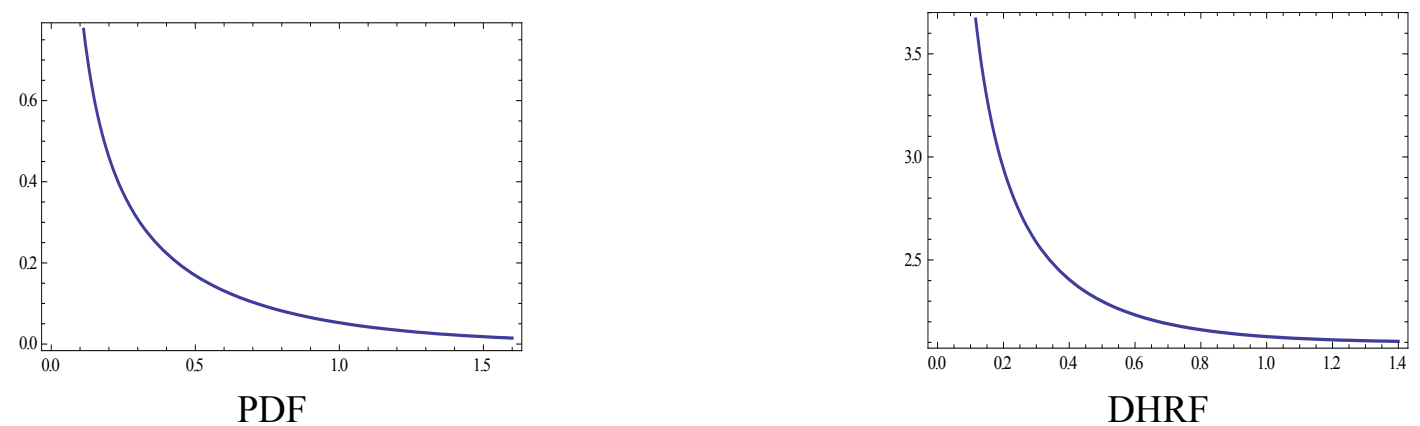

Fig. 1. Case a: $(\delta=0.2, \gamma=0.7, \beta=3)$

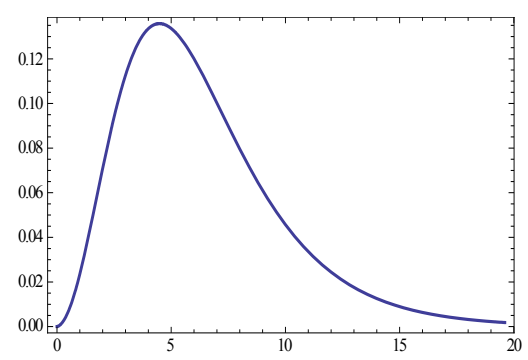

PDF

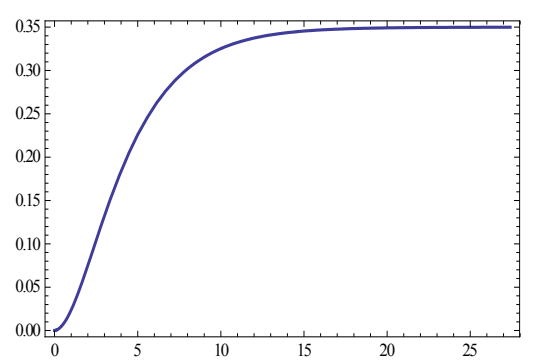

IHRF

Fig. 2. Case b: $(\delta=5.0, \gamma=0.7, \beta=0.5)$
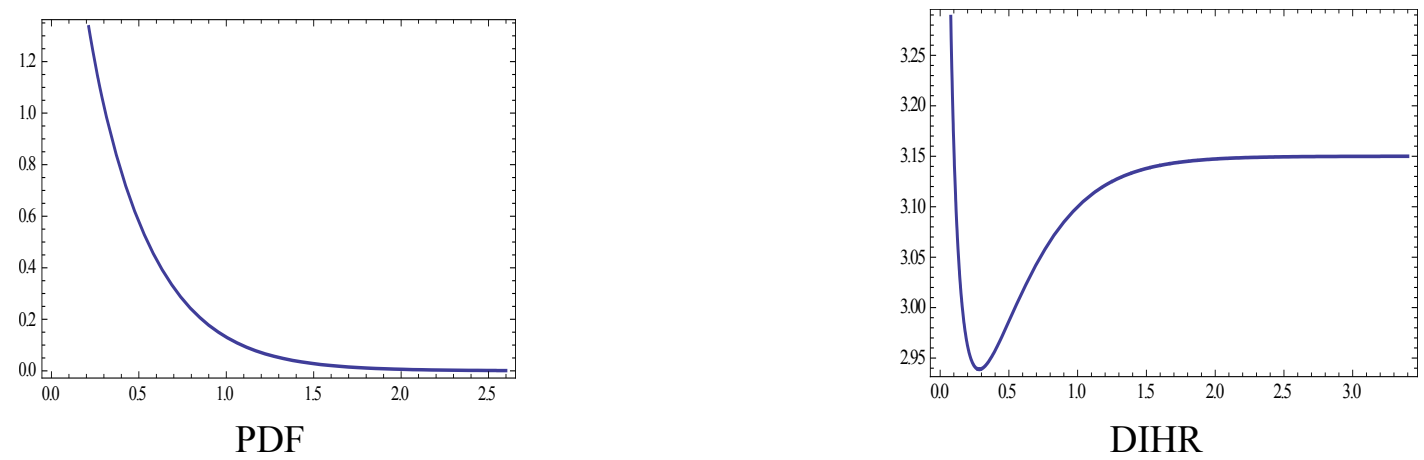

Fig. 3. Case c: $(\delta=1.0, \gamma=0.7, \beta=4.5)$
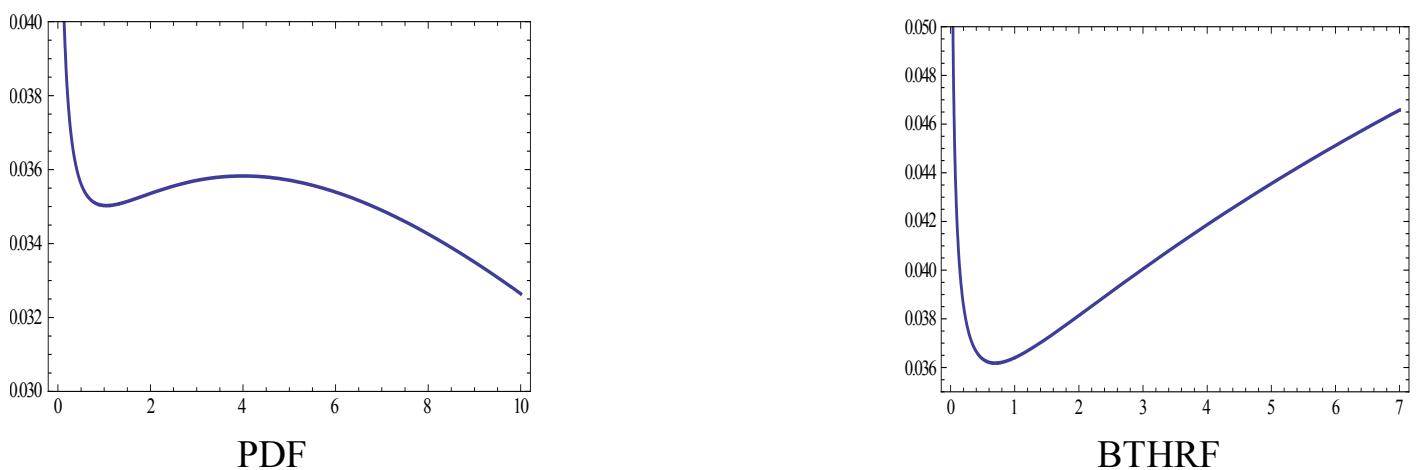

Fig. 4. Case d: $(\delta=1.8, \gamma=0.7, \beta=0.1)$ 

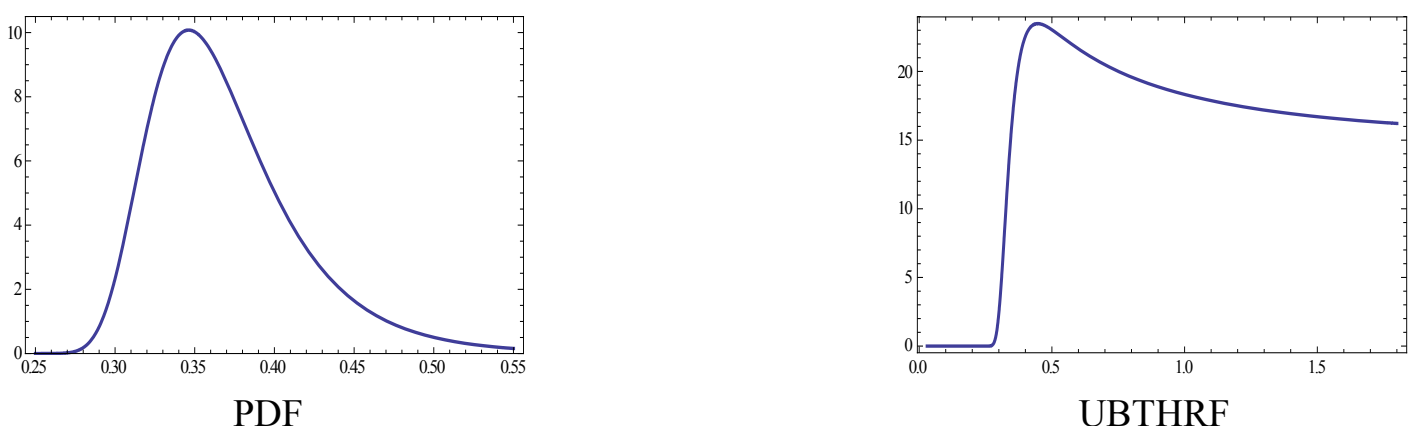

Fig. 5. Case e: $(\delta=0.4, \gamma=12.7, \beta=1.2)$

\section{Bayes One-Sample Interval Prediction of Future Observables}

Statistical prediction is the problem of inferring the values of unknown observables (future observations), or functions of such observables, from current available (informative) observations. A predictor could be a point or interval predictor. Frequentist and Bayesian methods have been used to obtain predictors and study their properties. "The problem of prediction can be solved fully within Bayes framework" Geisser [12]. "Inference about parameters is thus seen to be a limiting form of predictive inference about observables" Bernardo and Smith [13].

\subsection{One-sample scheme and subjective prior}

In the one-sample scheme, $X_{1}<\ldots<X_{r}$ represent the informative sample which are the first $r$ order statistics in a random sample of size $n$ drawn from a population whose CDF and PDF are $F(x)$ and $f(x)$, respectively. The future sample is represented by the rest of order statistics $X_{r+1}<\ldots<X_{n}$. The likelihood function (LF) is given by

$$
L^{*}(\theta, \mathrm{x}) \propto\left[\prod_{i=1}^{r} f\left(x_{i}\right)\right]\left[1-F\left(x_{r}\right)\right]^{n-r},
$$

where $\boldsymbol{\theta}=(\delta, \gamma, \beta)$ and $\mathbf{x}=\left(x_{1}, \ldots, x_{r}\right)$.

Suppose that the prior belief of the experimenter is measured by a PDF, given by

$$
\pi(\boldsymbol{\theta})=\pi_{1}(\delta) \pi_{2}(\gamma, \beta),
$$

where $\delta$ and $(\gamma, \beta)$ are independent such that

$$
\begin{gathered}
\pi_{1}(\delta) \propto \delta^{c_{1}-1} e^{-c_{2} \delta}, \delta>0,\left(c_{1}, c_{2}>0\right), \\
\pi_{2}(\gamma, \beta)=\pi_{21}(\gamma \mid \beta) \pi_{22}(\beta) \propto\left[\beta^{c_{3}} \gamma^{c_{3}-1} e^{-\beta \gamma}\right]\left[\beta^{c_{4}-1} e^{-c_{5} \beta}\right], \gamma, \beta>0,\left(c_{3}, c_{4}, c_{5}>0\right) .
\end{gathered}
$$

So that

$$
\pi(\boldsymbol{\theta}) \propto \delta^{c_{1}-1} \gamma^{c_{3}-1} \beta^{c_{3}+c_{4}-1} e^{-c_{2} \delta-\left(c_{5}+\gamma\right) \beta}, \delta, \gamma, \beta>0, c_{i}>0, i=1, \ldots, 5 .
$$

By combining (21) and (22), the posterior PDF is then given by

$$
\pi(\boldsymbol{\theta} \mid \mathbf{x}) \propto L(\boldsymbol{\theta}, \mathbf{x}) \pi(\boldsymbol{\theta}) \propto \pi(\boldsymbol{\theta})\left[1-F\left(x_{r}\right)\right]^{n-r}\left[\prod_{i=1}^{r} f\left(x_{i}\right)\right],
$$

where $\pi(\boldsymbol{\theta})$ is given by (22). 
Let $Y_{s}=X_{r+s}, s=1,2, \ldots, n-r$ and denote by $f_{r}\left(y_{s} \mid \theta\right)$ the PDF of the $s^{\text {th }}$ component to fail given that $r$ components had already failed. Then

$$
\begin{aligned}
f_{r}\left(y_{s} \mid \theta\right) & \propto\left[F\left(y_{s}\right)-F\left(x_{r}\right)\right]^{s-1}\left[1-F\left(y_{s}\right)\right]^{n-r-s}\left[1-F\left(x_{r}\right)\right]^{-(n-r)} f\left(y_{s}\right) \\
& \propto \sum_{j_{1}=0}^{s-1} \sum_{j_{2}=0}^{n-r-s} a_{j_{1}} a_{j_{2}}\left[F\left(y_{s}\right)\right]^{s-j_{1}+j_{2}-1}\left[F\left(x_{r}\right)\right]^{j_{1}}\left[1-F\left(x_{r}\right)\right]^{-(n-r)} f\left(y_{s}\right),
\end{aligned}
$$

where

$$
a_{j_{1}}=(-1)^{j_{1}}\left(\begin{array}{c}
s-1 \\
j_{1}
\end{array}\right), \quad a_{j_{2}}=(-1)^{j_{2}}\left(\begin{array}{c}
n-r-s \\
j_{2}
\end{array}\right) .
$$

The predictive PDF $f^{*}\left(y_{s} \mid x\right)$ of the $s^{\text {th }}$ future observable, given data, is defined by

$$
f^{*}\left(y_{s} \mid \mathbf{x}\right)=\int f_{r}\left(y_{s} \mid \boldsymbol{\theta}\right) \pi(\boldsymbol{\theta} \mid \mathbf{x}) d \mathbf{\theta} .
$$

Substitution of (23) and (24) in (26), then yields

$$
f^{*}\left(y_{s} \mid \mathbf{x}\right)=A \sum^{*} a^{*} \int \pi(\boldsymbol{\theta})\left[F\left(y_{s}\right)\right]^{s-j_{1}+j_{2}-1} f\left(y_{s}\right)\left[F\left(x_{r}\right)\right]^{j_{1}}\left[\prod_{i=1}^{r} f\left(x_{i}\right)\right] d \boldsymbol{\theta},
$$

where $A$ is a normalizing constant,

$$
\sum^{*}=\sum_{j_{2}=0}^{s-1} \sum_{j_{3}=0}^{n-r-s}, a^{*}=a_{j_{1}} a_{j_{2}}, \int \equiv \int_{0}^{\infty} \int_{0}^{\infty} \int_{0}^{\infty}, d \boldsymbol{\theta} \equiv d \delta d \gamma d \beta .
$$

The predictive SF $P\left[Y_{s}>v \mid \mathbf{x}\right]$ of $Y_{s}$ given data is then given by

$$
\begin{aligned}
& P\left[Y_{S}>v \mid \mathbf{x}\right]= \int_{v}^{\infty} f^{*}\left(y_{S} \mid \mathbf{x}\right) d y_{S} \\
&= A \sum^{*} a^{*} \int \pi(\boldsymbol{\theta})\left[F\left(x_{r}\right)\right]^{j_{1}}\left[\prod_{i=1}^{r} f\left(x_{i}\right)\right] \int_{v}^{\infty}\left[F\left(y_{s}\right)\right]^{s-j_{1}+j_{2}-1} f\left(y_{s}\right) d y_{s} d \boldsymbol{\theta} \\
&= A \sum^{*}\left(\frac{a^{*}}{s-j_{1}+j_{2}}\right) \int \pi(\boldsymbol{\theta})\left\{1-[F(v)]^{s-j_{1}+j_{2}}\right\}\left[F\left(x_{r}\right)\right]^{j_{1}}\left[\prod_{i=1}^{r} f\left(x_{i}\right)\right] d \boldsymbol{\theta} \\
& \Rightarrow \quad P\left[Y_{s}>v \mid \mathbf{x}\right]=A S(v), v>x_{r}, \quad S(v)=\sum^{*}\left(\frac{a^{*}}{s-j_{1}+j_{2}}\right) I(v), \\
& I(v)=\int_{0}^{\infty} \pi(\boldsymbol{\theta})\left\{1-[F(v)]^{s-j_{1}+j_{2}}\right\}\left[F\left(x_{r}\right)\right]^{j_{1}}\left[\prod_{i=1}^{r} f\left(x_{i}\right)\right] d \boldsymbol{\theta} .
\end{aligned}
$$


Since $1=P\left[Y_{s}>x_{r} \mid x\right]=A S\left(x_{r}\right)$, then $A=\frac{1}{S\left(x_{r}\right)}$. Therefore, from (29),

$$
P\left[Y_{s}>v \mid x\right]=\frac{S(v)}{S\left(x_{r}\right)}, v>x_{r}
$$

where $S($.$) is given by (30) in which I(v)$ is given by (31).

Substitution of $\pi(\boldsymbol{\theta}), F($.$) and f($.$) , given by (22), (7) and (8), in (31), yields$

$$
\begin{aligned}
I(v)= & \int_{0}^{\infty} \int_{0}^{\infty} \int_{0}^{\infty} \delta^{c_{1}-1} \gamma^{c_{3}-1} \beta^{c_{3}+c_{4}-1} e^{-c_{2} \delta-\left(c_{5}+\gamma\right) \beta} \\
& \left\{1-\left[\exp \left\{-\delta\left(s-j_{1}+j_{2}\right)\left(-\ln \left(1-e^{-\beta v}\right)\right)^{\gamma}\right\}\right]\right\} \exp \left\{-\delta j_{1}\left(-\ln \left(1-e^{-\beta x_{r}}\right)\right)^{\gamma}\right\} \\
& {\left[\prod_{i=1}^{r}\left\{\delta \gamma \beta\left(\frac{e^{-\beta x_{i}}}{1-e^{-\beta x_{i}}}\right)\left(-\ln \left(1-e^{-\beta x_{i}}\right)^{\gamma}\right\} \exp \left\{-\delta\left(-\ln \left(1-e^{-\beta x_{i}}\right)\right)^{\gamma}\right\}\right] d \delta d \gamma d \beta .\right.} \\
\Rightarrow \quad & I(v)=\int_{0}^{\infty} \int_{0}^{\infty} Q(\beta, \gamma) I_{v}(\beta, \gamma) d \beta d \gamma,
\end{aligned}
$$

where

$$
\begin{gathered}
Q(\beta, \gamma)=\gamma^{r+c_{3}-1} \beta^{r+c_{3}+c_{4}-1} e^{-\beta\left(c_{5}+\gamma\right)} \prod_{i=1}^{r}\left\{\left(\frac{e^{-\beta x_{i}}}{1-e^{-\beta x_{i}}}\right)\left(-\ln \left(1-e^{-\beta x_{i}}\right)\right)^{\gamma-1}\right\}, \\
I_{v}(\beta, \gamma)=\int_{0}^{\infty} \delta^{r+c_{1}-1}\left[e^{-B\left(x_{r}\right) \delta}-e^{-C(v) \delta}\right] d \delta \\
=\Gamma\left(r+c_{1}\right)\left\{\left[B\left(x_{r}\right)\right]^{-\left(r+c_{1}\right)}-[C(v)]^{-\left(r+c_{1}\right)}\right\} . \\
B\left(x_{r}\right)=c_{2}+j_{2}\left(-\ln \left(1-e^{-\beta x_{r}}\right)\right)^{\gamma}+\sum_{i=1}^{r}\left(-\ln \left(1-e^{-\beta x_{i}}\right)\right)^{\gamma}, \\
C(v)=B\left(x_{r}\right)+\left(s-j_{1}+j_{2}\right)\left\{-\ln \left(1-e^{-\beta v}\right)\right\}^{\gamma} .
\end{gathered}
$$

Substitution of (34) and (35) in (33) then yields

$$
I(v)=\Gamma\left(r+c_{1}\right) \int_{0}^{\infty} \int_{0}^{\infty} Q(\beta, \gamma)\left\{\left[B\left(x_{r}\right)\right]^{-\left(r+c_{1}\right)}-[C(v)]^{-\left(r+c_{1}\right)}\right\} d \beta d \gamma
$$

The lower and upper bounds $L$ and $U$ of $100(1-\tau) \%$ prediction intervals for the $s^{\text {th }}$ future observable $Y_{s}, s=r+1, \ldots, n$ are given by the solution of the two equations

$$
P\left[Y_{s}>L \mid x\right]=1-\frac{\tau}{2} \text { and } P\left[Y_{s}>U \mid x\right]=\frac{\tau}{2} .
$$

Equivalently, $L$ and $U$ are computed by the use of (32) from the two equations

$$
\left.\begin{array}{l}
S(L)-(1-\tau / 2) S\left(x_{r}\right)=0, \\
S(U)-(\tau / 2) S\left(x_{r}\right)=0 .
\end{array}\right\}
$$




\section{Bayes Two-Sample Prediction of Future Obsrvables}

Two samples are considered. The informative sample is given (as in the one-sample scheme) by the first $r$ order statistics $X_{1}<\ldots<X_{r}$ of a random sample of size $n$ drawn from a population whose CDF is given by (17). It is assumed that the future sample is given by another set of order statistics $Y_{1}<\ldots<Y_{m}$, of a random sample of size $m$, independent of the first sample, drawn from the same population. We wish to predict $Y_{s}, s=1, \ldots, m$. In this case, the posterior PDF is given by (23). The predictive PDF $f^{*}\left(y_{s} \mid \mathbf{x}\right)$ is given by (27), with $f_{r}\left(y_{s} \mid \boldsymbol{\theta}\right)$ being replaced by the PDF $h\left(y_{s} \mid \boldsymbol{\theta}\right)$ of the $s^{\text {th }}$ order statistic in a sample of size $m$

$$
\begin{gathered}
h\left(y_{s} \mid \boldsymbol{\theta}\right) \propto\left[F\left(y_{s}\right)\right]^{s-1}\left[1-F\left(y_{s}\right)\right]^{m-s} f\left(y_{s}\right) \\
\propto \sum_{j_{2}=0}^{m-s} a_{j_{2}}^{*}\left[F\left(y_{s}\right)\right]^{s+j_{2}-1} f\left(y_{s}\right) .
\end{gathered}
$$

LF (21) can be written as

$$
L^{*}(\theta, \mathrm{x}) \propto\left[\prod_{i=1}^{r} f\left(x_{i}\right)\right] \sum_{j_{1}=0}^{n-r} a_{j_{1}}\left[F\left(x_{r}\right)\right]^{j_{1}} .
$$

So that the predictive PDF $f_{1}^{*}\left(y_{s} \mid \mathbf{x}\right)$ of the $s^{\text {th }}$ future observable, is given by

$$
f_{1}^{*}\left(y_{s} \mid \mathbf{x}\right)=A_{1} \sum_{j_{1}=0}^{n-r} \sum_{j_{2}=0}^{m-s} a_{j_{1}}^{*} a_{j_{2}}^{*} \int \pi(\boldsymbol{\theta})\left[F\left(x_{r}\right)\right]^{j_{1}}\left[\prod_{i=1}^{r} f\left(x_{i}\right)\right]\left[F\left(y_{s}\right)\right]^{s+j_{2}-1} f\left(y_{s}\right) d \boldsymbol{\theta},
$$

where $A_{1}$ is a normalizing constant, $\pi(\boldsymbol{\theta})$ is given by (22), $a_{j_{1}}^{*}$ and $a_{j_{2}}^{*}$ by

$$
a_{j_{1}}^{*}=(-1)^{j_{1}}\left(\begin{array}{c}
n-r \\
j_{1}
\end{array}\right), \quad a_{j_{2}}^{*}=(-1)^{j_{2}}\left(\begin{array}{c}
m-s \\
j_{2}
\end{array}\right),
$$

$\int$ and $d \boldsymbol{\theta}$ by (28).

By substituting $\pi(\boldsymbol{\theta}), F($.$) and f($.$) , given by (22), (7) and (8) and simplifying, it can be shown that the$ predictive SF is given by

$$
P_{1}^{*}\left[Y_{s}>v \mid \mathrm{x}\right]=\frac{S_{1}^{*}(v)}{S_{1}^{*}(0)}, \quad v>0
$$

where

$$
S_{1}^{*}(v)=\sum_{j_{1}=0}^{n-r} \sum_{j_{2}=0}^{m-s} \frac{a_{j_{1}}^{*} a_{j_{2}}^{*}}{s+j_{2}} I^{*}(v)
$$

$I^{*}(v)$ is of the form (33) in which $Q^{*}(\beta, \gamma)$ and $I_{v}^{*}(\beta, \gamma)$ are of the same form as (34) and (35), except that

$$
B^{*}\left(x_{r}\right)=c_{2}+j_{1}\left\{-\ln \left(1-e^{-\beta x_{r}}\right)\right\}^{\gamma}+\sum_{i=1}^{r}\left\{-\ln \left(1-e^{-\beta x_{i}}\right)\right\}^{\gamma},
$$

and

$$
C^{*}(v)=B^{*}\left(x_{r}\right)+\left(s+j_{2}\right)\left\{-\ln \left(1-e^{-\beta v}\right)\right\}^{\gamma}
$$


It may be noticed that

$$
S_{1}^{*}(0)=\sum_{j_{1}=0}^{n-r} \sum_{j_{2}=0}^{m-s} \frac{a_{j_{1}}^{*} a_{j_{2}}^{*}}{s+j_{2}} I^{*}(0)
$$

where $I^{*}(0)$ is of the form (33), in which $I_{0}(\beta, \gamma)=\Gamma\left(r+c_{1}\right)\left[B^{*}\left(x_{r}\right)\right]^{-\left(r+c_{1}\right)}$, where $B^{*}\left(x_{r}\right)$ is given by (44).

The lower and upper bounds, $L$ and $U$, of $100(1-\tau) \%$ prediction interval are computed by the use of (42) from the two equations

$$
\left.\begin{array}{l}
S_{1}^{*}(L)-(1-\tau / 2) S_{1}^{*}(0)=0, \\
S_{1}^{*}(U)-(\tau / 2) S_{1}^{*}(0)=0 .
\end{array}\right\}
$$

\section{Examples and Numerical Computations}

This section consists of two parts: real data analysis in which a comparison is made between the WGEE and Weibull models, to illustrate the method. The other part is a simulation study to measure the credibility of results.

\subsection{Example 1: Real lifetime data: A comparison between the WGEE and Weibull models}

The following observations represent time-to-failure (in months) of 20 electronic components on test, see Wingo [14],

$$
\begin{array}{llllllllll}
0.1, & 0.1, & 0.2, & 0.3, & 0.4, & 0.5, & 0.5, & 0.6, & 0.7, & 0.8 \\
0.9, & 0.9, & 1.2, & 1.6, & 1.8, & \mathbf{2 . 3}, & \mathbf{2 . 5}, & \mathbf{2 . 6}, & \mathbf{2 . 9}, & \mathbf{3 . 1}
\end{array}
$$

The WGEE and $\mathrm{W}(a, b)$, whose CDFs are given by (7) and $F(x)=1-\exp \left(-a x^{b}\right)$ are fitted to the set of data. In Table 1, the MLEs of the parameters, Kolmogorov Smirnov test statistics and p-values are computed, using the above data, when the underlying distribution is Weibull as compared with the WGEE.

Table 1. MLEs, K-S Statistics and p-Values for W and WGEE

\begin{tabular}{|c|c|c|c|}
\hline & MLEs & K-S & p-Value \\
\hline $\mathrm{W}$ & $\hat{a}_{M L}=0.74789, \hat{b}_{M L}=1.19626$ & 0.11719 & 0.94407 \\
\hline WGEE & $\hat{\delta}_{M L}=1.09568, \hat{\gamma}_{M L}=1.14412, \hat{\beta}_{M L}=0.80804$ & 0.09405 & 0.96277 \\
\hline
\end{tabular}

Fig. 6 shows the graphs of PDF and HRF of the WGEE distribution, when the parameters are replaced by their MLEs: $\hat{\delta}=1.09568 \hat{\gamma}=1.14412$ and $\hat{\beta}=0.80804$.

Notice that this shape of HRF is not accommodated by the Weibull distribution.

Based on Kolmogorov-Smirnov (K-S) statistics and p-values, the WGEE distribution fits better than the Weibull distribution. This is also shown by plotting the empirical CDF versus the CDFs of $\operatorname{WGEE}\left(\hat{\delta}_{M L}, \hat{\gamma}_{M L}, \hat{\beta}_{M L}\right)$ and $\mathrm{W}\left(\hat{a}_{M L}, \hat{b}_{M L}\right)$, see Fig. 7 . 

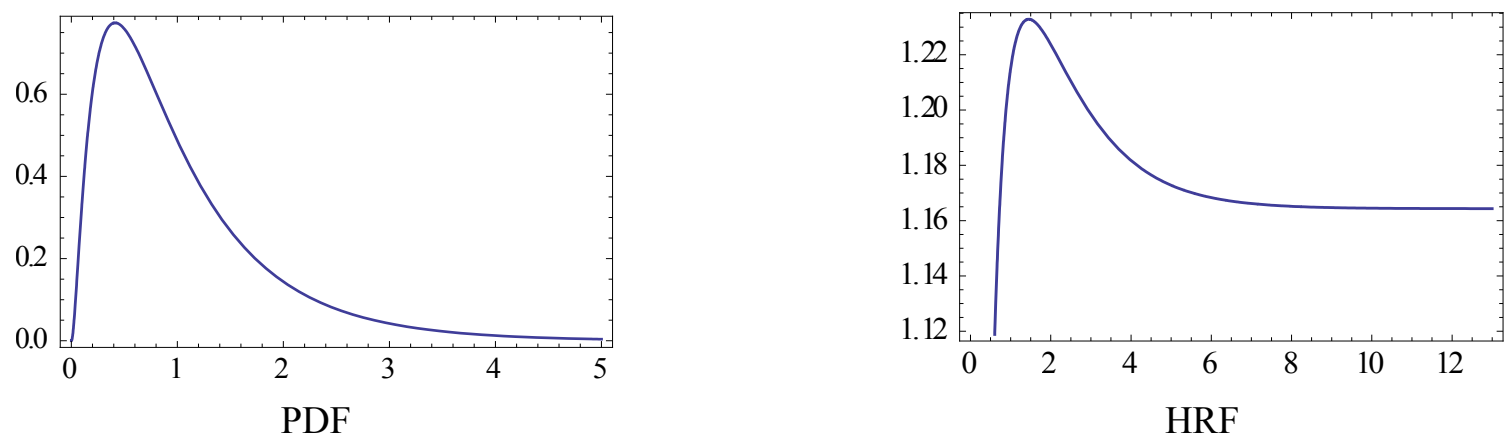

Fig. 6. The PDF and HRF

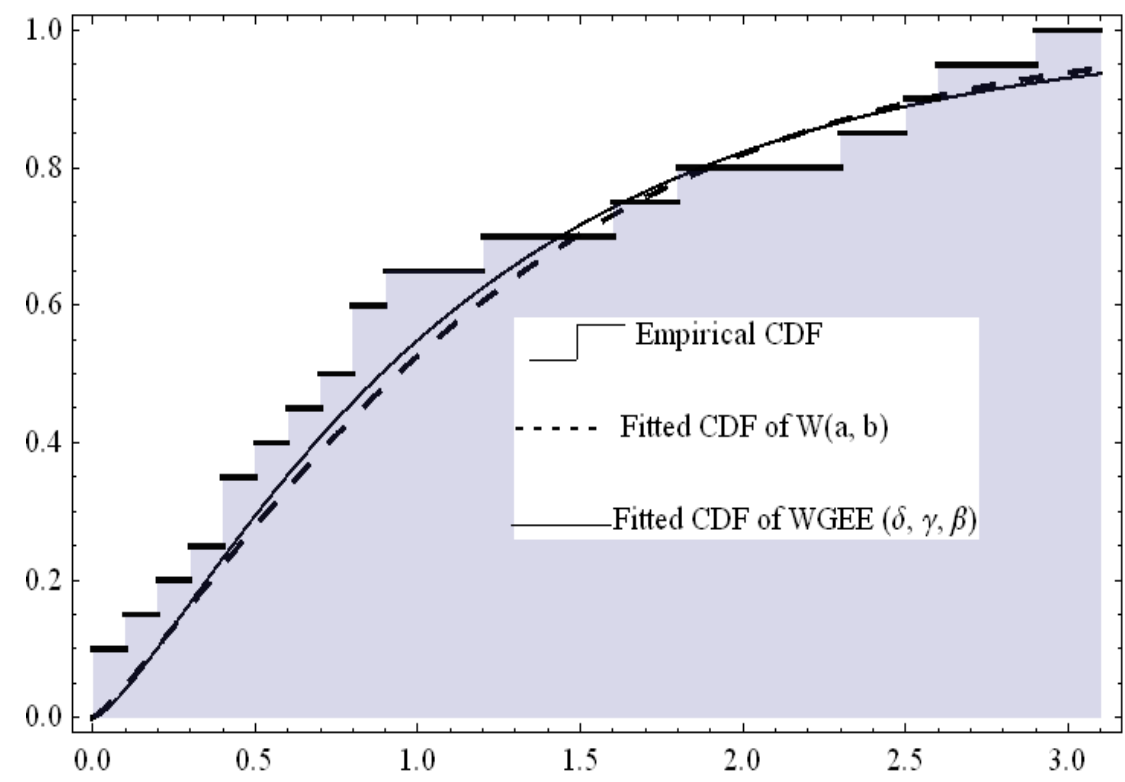

Fig. 7. Empirical CDF versus CDFs of $\mathrm{W}$ and WGEE

Table 2. $95 \%$ Prediction Intervals of Future Observables $Y_{s}$ From

$\operatorname{WGEE}(\hat{\delta}=1.09568, \hat{\gamma}=1.14412, \hat{\beta}=0.80804)$, Based on One-Sample Scheme $n=20, r=15$ $c_{1}=1.07, c_{2}=0.97, c_{3}=0.93, c_{4}=1.1, c_{5}=1.35$.

\begin{tabular}{ccc}
\hline$Y_{s}$ & Prediction Interval & Interval Length \\
\hline$s=1$ & $(1.80805,3.12377)$ & 1.31572 \\
$s=2$ & $(1.72430,4.26280)$ & 2.53850 \\
$s=3$ & $(2.05588,5.63120)$ & 3.57532 \\
$s=4$ & $(2.33040,7.74559)$ & 5.41519 \\
$s=5$ & $(2.82306,12.4550)$ & 9.62198
\end{tabular}


Table 2 displays the lower and upper bounds $L$ and $U$ of the $95 \%$ prediction intervals of the future observables $Y_{s}=X_{r+s}, s=1, \ldots 5, r=15, n=20$, from WGEE, in the one-sample case. The hyperparameters are assigned the values:

$$
c_{1}=1.07, c_{2}=0.97, c_{3}=0.93, c_{4}=1.1, c_{5}=1.35 .
$$

Eqs. (39) are solved for $L$ and $U$.

To study the effect of the future sample size, in the two-sample case, Table 3 displays the lower and upper bounds $L$ and $U$ of the $95 \%$ two- sample prediction intervals of the future observables $Y_{s}$, for different values of s, $n=20, r=15 m=10,15,20$, from WGEE $\left(\hat{\delta}_{M L}=1.09568, \hat{\gamma}_{M L}=1.14412, \hat{\beta}_{M L}=0.80804\right)$. The hyper-parameters are assigned the values:

$$
c_{1}=1.07, c_{2}=0.97, c_{3}=0.93, c_{4}=1.1, c_{5}=1.35 .
$$

Equations (46) are solved for $L$ and $U$.

Table 3. 95\% Two-sample Prediction Intervals of Future Observables $Y_{s}$, From

$$
\begin{gathered}
\operatorname{WGEE}(\hat{\delta}=1.09568, \hat{\gamma}=01.14412, \hat{\beta}=0.80804) \\
n=20, r=15, m=10,15,20 \text { and } \\
c_{1}=1.07, c_{2}=0.97, c_{3}=0.93, c_{4}=1.1, c_{5}=1.35 .
\end{gathered}
$$

\begin{tabular}{cccc}
\hline$m$ & $s$ & Prediction interval & Length \\
\hline 10 & 1 & $\left(6.228 \times 10^{-6}, 0.68548\right)$ & 0.68548 \\
& 5 & $(0.16594,3.23172)$ & 3.06578 \\
& 10 & $(1.31927,6.19979)$ & 4.88052 \\
15 & 1 & $\left(1.543 \times 10^{-6}, 0.41983\right)$ & 0.41983 \\
& 8 & $(0.28342,4.06005)$ & 3.77663 \\
& 15 & $(1.66002,5.69787)$ & 4.03785 \\
20 & 1 & $\left(5.434 \times 10^{-7}, 0.29582\right)$ & 0.29582 \\
& 10 & $(0.37476,4.25630)$ & 3.88154 \\
& 20 & $(1.90577,6.08348)$ & 4.17771
\end{tabular}

\subsection{Simulation}

\subsubsection{Example 2: (One-sample scheme)}

The following steps are followed to compute the lower and upper bounds of the prediction intervals of future observables. 
(1) For given $(\delta, \gamma, \beta)$, generate $n$ observations from $\operatorname{WGEE}(\delta, \gamma, \beta)$, given by (7), according to

$$
X=-\frac{1}{\beta} \ln \left[1-\exp \left\{-\left[-\frac{\ln U}{\delta}\right]^{1 / \gamma}\right\}\right],
$$

where $U$ is uniform on the interval $(0,1)$.

(2) Order data and censor at $r$.

(3) Compute $L$ and $U$, such that, for given $\tau=0.05$, they satisfy (39), where $S(v)$ is given by (30).

A random sample of size $n=20$ is generated according to step 1 , when $(\delta=5, \gamma=0.7, \beta=0.5)$ and then ordered, with $r=15$, is given by

$1.88714,3.13577,3.26199,3.46851,3.57868,3.65395,3.67780,4.26213,4.41463,4.46442,4.63356$,

4.74583, 4.86271, 5.07773, 6.63053, 7.21648, 7.55987, 7.70941, 10.76840, 14.075.

The first $r=15$ ordered observations represent the informative sample. The lower and upper bounds are computed by solving Eqs. (39).

Table 4 shows the $95 \%$ prediction intervals for the future observables $Y_{s} \equiv X_{r+s}, s=1, \ldots, 5, r=15$, in the one-sample case. The hyper-parameters are assigned the values:

$$
c_{1}=3.0, c_{2}=0.6, c_{3}=0.35, c_{4}=1.4, c_{5}=2.8 \text {. }
$$

Table 4. $95 \%$ One-Sample Prediction Intervals of Future Observables $Y_{s}$ From

$\operatorname{WGEE}(\delta=5, \gamma=0.7, \beta=0.5), n=20, r=15$

\begin{tabular}{ccc}
\hline$Y_{s}$ & Prediction Interval & Interval Length \\
\hline$s=1$ & $(6.65449,9.95246)$ & 3.29798 \\
$s=2$ & $(6.88167,12.1342)$ & 5.25248 \\
$s=3$ & $(7.34655,14.7770)$ & 7.43045 \\
$s=4$ & $(8.08966,18.7283)$ & 10.6386 \\
$s=5$ & $(9.36474,27.4809)$ & 18.1162 \\
\hline
\end{tabular}

\subsubsection{Simulation results (One-sample case)}

The above Example 1 is based on one sample. The computations are repeated for 500 samples. The coverage probabilities (COVP) and average interval lengths (AIL) of the $95 \%$ prediction intervals of $x_{r+s}, s=1, \ldots, 5$ over 500 samples, each of size $n=20$, censored at $r=15$, from $\operatorname{WGEE}(\delta=5, \gamma=0.7, \beta=0.5)$ are reported in Table 5.

Table 5. COVP and AIL for 500 Samples.

\begin{tabular}{cccccc}
\hline & $\mathrm{s}=1$ & $\mathrm{~s}=2$ & $\mathrm{~s}=3$ & $\mathrm{~s}=4$ & $\mathrm{~s}=5$ \\
\hline COVP & 95.1 & 95.0 & 96.8 & 97.0 & 96.3 \\
AIL & 3.6153 & 5.9037 & 8.7260 & 12.0844 & 22.1819 \\
\hline
\end{tabular}




\subsubsection{Example 3: Simulation results (Two-sample case)}

In this example, two independent samples of sizes $n$ and $m$ are assumed to be drawn from WGEE ( $\delta=5, \gamma=0.7, \beta=0.5)$ distribution. The informative sample and hyper-parameters are chosen to be the same as in the one-sample case, given in Example 1. Table 6 shows Bayes prediction intervals of future observables $Y_{S}$, using the two-sample scheme, where $m=10,15,20, n=20, r=15$.

Table 6. 95\% Prediction Intervals of Future Observables $Y_{s}$ From $\operatorname{WGEE}(\delta=5, \gamma=0.7, \beta=0.5)$, Based on Two-Sample Scheme, $n=20, r=15, m=10,15,20$,

$$
c_{1}=3.0, c_{2}=0.6, c_{3}=0.35, c_{4}=1.4, c_{5}=2.8 \text {. }
$$

\begin{tabular}{ccccc}
\hline$m$ & $s$ & Prediction Interval & Length & COVP \\
\hline 10 & 1 & $(0.53355,4.15904)$ & 3.62549 & 95.594 \\
& 5 & $(2.79011,8.45758)$ & 5.66747 & 98.336 \\
& 10 & $(6.95511,18.2622)$ & 11.30709 & 90.748 \\
15 & 1 & $(0.43981,3.58604)$ & 3.14623 & 96.348 \\
& 8 & $(3.37532,8.49993)$ & 5.12461 & 98.760 \\
& 15 & $(8.50800,19.8057)$ & 11.2977 & 90.554 \\
& 1 & $(0.38123,3.25463)$ & 2.87340 & 96.804 \\
& 10 & $(3.27221,7.89192)$ & 4.61971 & 99.086 \\
& 20 & $(9.21162,20.4685)$ & 11.25688 & 90.722 \\
\hline
\end{tabular}

\section{Concluding remarks}

We have considered in this paper the WGEED a new model which arises as a composition of Weibull and exponentiated exponential distribution. The new model has several shapes of the HRF. Prediction intervals for future observables are obtained using one- and two-sample schemes. The following remarks may be observed:

- The CDF (7) of the WGEE is obtained in closed form that simplifies its use.

- Generation of cumulative distribution functions by composition with other cumulative distributions or functions of such distributions can add at least an extra parameter to a distribution. The 3-parameter WGEE model gives a better fit than the 2-parameter Weibull model. This extra parameter makes it more flexible to fitting data. Notice the five different shapes of the HRFs of the WGEE model.

- In either one- or two-sample cases, the lengths of intervals increase by increasing the index $s$ of the future observable $y_{s}$ (Table 5 and Table 6).

- In the one-sample case, the confidence interval of the first future observable $Y_{1}$ is the best (shortest length) among all future observables (Table 5). 
- In the two-sample case, the lengths of intervals decrease by increasing the future sample size $m$ (Table 6).

- It may be noticed that the coverage probabilities approach the nominal confidence level of $95 \%$ in both sample schemes.

- It should be pointed out that if the hyper-parameters are unknown, we may use the empirical Bayes method to estimate them using past samples, see Maritz and Lwin [14]. Alternatively, one could use the hierarchical Bayes method in which a suitable prior for the hyper-parameters is used [13].

- It is recommended to use the method when the variability in data is not too large. It has been found, using several samples, that large variability in data could lead to wide intervals.

\section{References}

[1] N. Eugene, C. Lee and F. Famoye, Beta-normal distribution and its applications, Commun. Statist - Th. Meth. 31 (2002) 497512.

[2] S. Nadarajah and A. K. Gupta, The beta Fréchet distribution, Far East J. Th. Statist.14 (2004) 15-24.

[3] S. Nadarajah and S. Kotz, The beta Gumble distribution, Math. Prob. Eng. 10 (2004) 323-332.

[4] S. Nadarajah and S. Kotz, The beta exponential distribution, Rel. Eng. Syst. Safety 91 (2006) 689-697.

[5] W. Barreto-Souza, A. H. S. Santos and G. M. Cordeiro, The beta generalized exponential distribution, J. Statist. Comput. Simul. 80 (2010) 159-172.

[6] P. F. Paranoíba, E. M. M. Ortega, G. M. Cordeiro and R. R. Pescim, The beta Burr XII distribution with application to life data, Comput. Statist. Data Anal. 55 (2011) 1118-1136.

[7] G. M. Cordeiro, A. B. Simas and B. D. Stošić, Closed form expressions for moments of the beta Weibull distribution, Anais da Academia Brasileira de Ciências 83(2) (2011) 357-373.

[8] G. M. Cordeiro and R. Brito, The beta power distribution, Brazilian J. Prob. Statist. 26 (2012) 88-112.[9] M. Ristić and N. Balakrishnan, An exponentiated exponential distribution generated by gamma random variables, J. Statist. Comput. Simul. 82(8) (2012) 1191-1206.

[9] Jones, M.C. (2004). Families of distributions arising from distributions of order statistics (with discussion). Test 13, 1-43.

[10] E. K. AL-Hussaini and M. Hussein, Estimation using censored data from exponentiated Burr type XII population, Open J. Statist.1 (2011). 13-45.

[11] S. Geisser, Predictive Inference: an Introduction (Chapman and Hall, London 1993).

[12] J. Bernardo and A. Smith, Bayesian Theory (Wiley, New York, 1994.

[13] D. R. Wingo, Maximum likelihood estimation of the Burr XII parameters under type II censoring, Microelectron. Reliab. 33 (1993) $1251-1257$.

[14] J. S. Maritz and T. Lwin, Empirical Bayes Methods, $2^{\text {nd }}$ edn. (Chapman \& Hall, London, 1989). 